\title{
Spectrum Imaging Techniques Applied to Forensics Investigations.
}

\author{
L.N. Brewer, P.G. Kotula, J.A. Ohlhausen, J.R. Michael
}

Sandia National Laboratories, Materials Characterization Dept., P.O. Box 5800-MS 1411 Albuquerque, NM 87185-1411

Unlike most materials characterization efforts, forensics samples come with little or no prior knowledge to aid the analyst in determining what techniques to use. The analyst needs to be able to compare large numbers of samples and answer the questions of 1.) Are these samples different?, 2.) How are they different?, and 3.) How different are they?

Spectrum imaging is ideally suited to addressing forensics analyses. Our approach is to combine a set of inorganic and organic analysis techniques to probe for compositional, molecular, and structural signals at the macro (bulk), micro, and nano length scales. Spectrum imaging techniques combined with multivariate statistical analysis allow for the collection and interrogation or enormous quantities of data without pre-biasing the answer.[1] Spectrum imaging has been used successfully in EDX microanalysis[1] (both in the SEM and TEM) and TOF-SIMS[2, 3].

In this study, a set of test biological agents, $\gamma$-irradiated Bacillus thuringiensis, were examined using a suite of spectrum imaging techniques. The sample set included a number of processing conditions to gauge the ability of these techniques to correctly identify the growth and weaponizing constituents present in the samples. SEM-EDX, STEM-EDX, and TOF-SIMS were used to examine each sample, representing a wide swath of data from the micro to nano scale and from $1 \mathrm{wt} \%$ sensitivity to ppm sensitivity.

The importance of combining data from multiple length scales and analytical sensitivities is highlighted in this study. Figures 1 and 2 compare data from the same sample at multiple length scales. In the SEM data (Figure 1), clumps of spore material with a large Si signal are observed, but no further detail is available as to the nature of the $\mathrm{Si}$ in this sample; however, individual $\mathrm{NaCl}$ particles are clearly observed. The same sample in the STEM demonstrates that the Si signal comes from nanoscale silica particles surrounding the spore with nearly nanoscale calcium phosphate particles also clinging to the particle (Figure 2). The $\mathrm{NaCl}$ signal is now only associated with the spore and is not observed in particulate form. If either data set was neglected, the complete attributes of this sample would have been missed. In another example, TOF-SIMS measurements were able to detect $\mathrm{Al}$ and $\mathrm{K}$ signals that were just observable by SEM-EDX (Figure 3A\&3B). The Sn signal in TOF-SIMS was measurable but was completely missed in EDX (Figure 3C). In addition, the combined analysis of positive and negative polarity spectra associated the $\mathrm{Cu}$ and $\mathrm{Cl}$ signals together as copper chloride particles.

1. P. G. Kotula, et al., Microscopy and Microanalysis. 9(1):(2003) p. 1-17.

2. J. A. Ohlhausen, et al., Applied Surface Science. 231-232:(2004) p. 230-234.

3. N. B. Gallagher, et al., Chemometrics and Intelligent Laboratory Systems. 73:(2004) p. 105117.

4. Sandia is a multiprogram laboratory operated by Sandia Corporation, a Lockheed Martin Company, for the United Stated Department of Energy (DOE) under contract DEAC0494AL85000. 
Figure 1. Comparison of data taken at multiple length scales. A.) SEM-BSE image of spore containing material, B.) Abundance map, and C.) EDX spectral components-red corresponds to clumps of spores, cyan corresponds to residual $\mathrm{NaCl}$ particles.

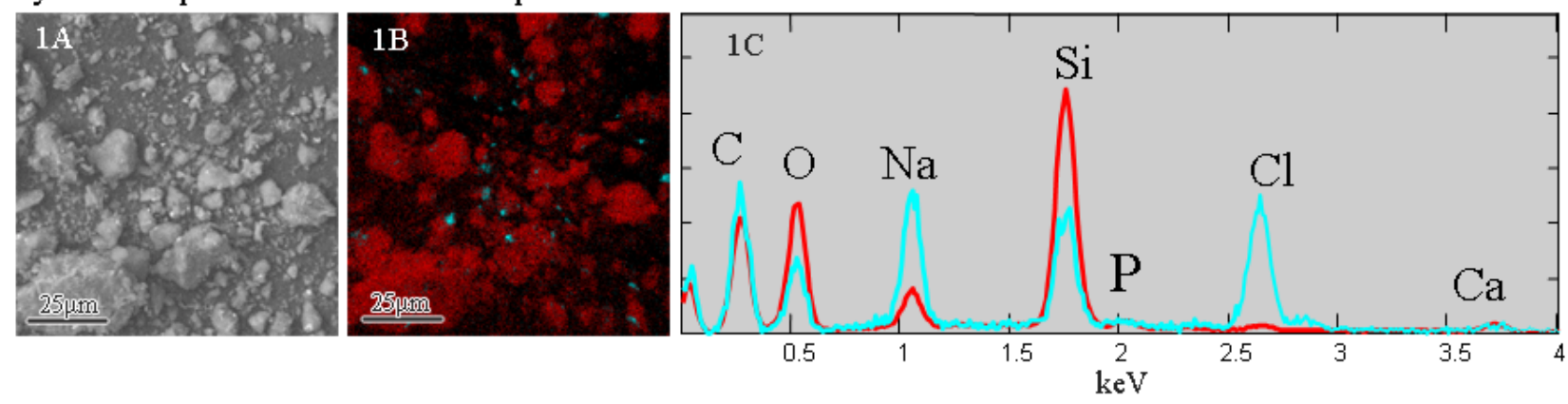

Figure 2. A.) STEM-ADF image of spore with particulates,

B.) Abundance map, and C.) EDX spectral components-red corresponds to spore, green corresponds to weaponizing agent, blue corresponds to residual growth media.
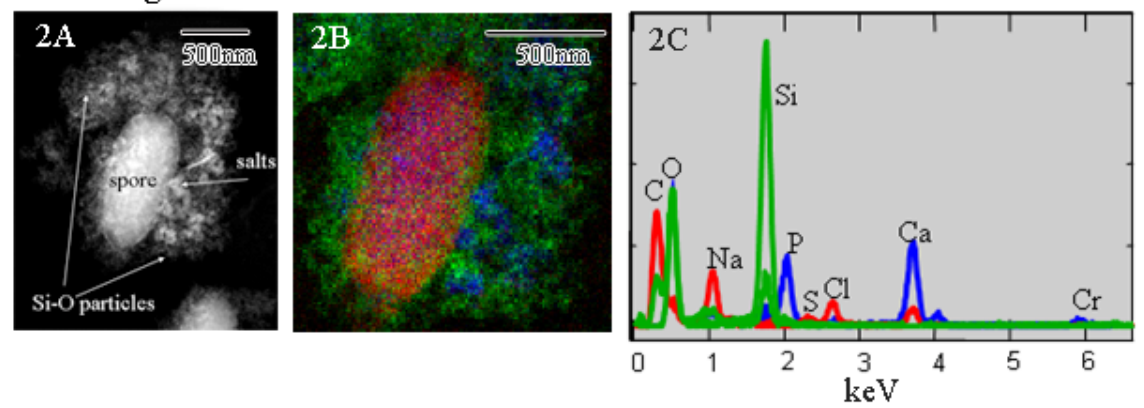

Figure 3. A.) Spectral components from TOF-SIMS data showing great analytical sensitivity to different metallic species, B.) Abundance map, and C.) expansion of spectral region around $\mathrm{Sn}$ signal.
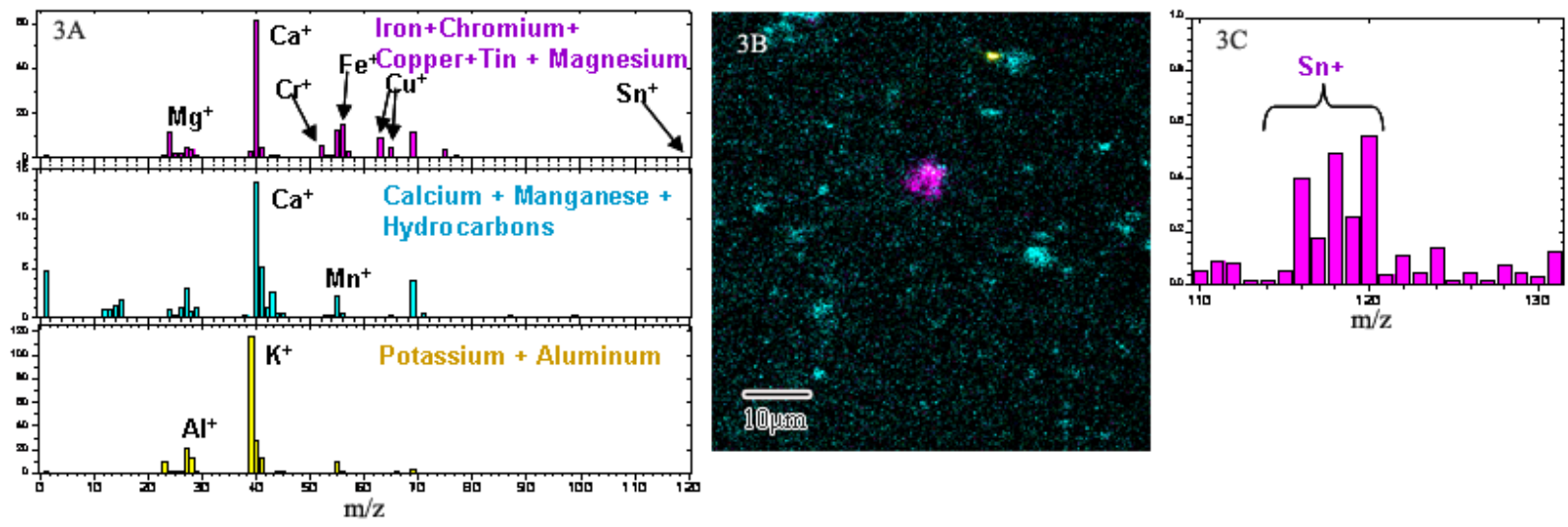